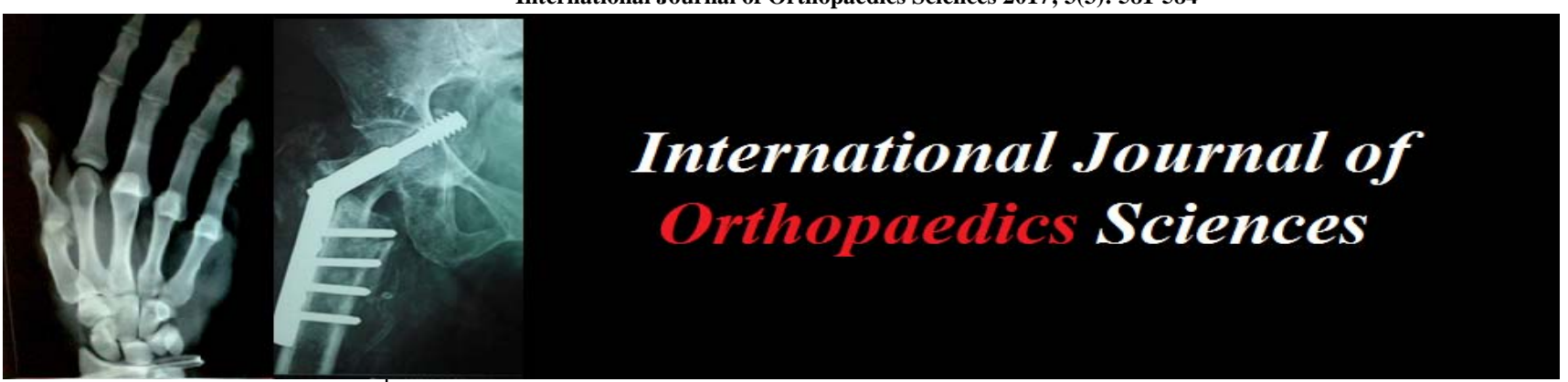

ISSN: $2395-1958$

IJOS 2017; 3(3): 581-584

(C) 2017 IJOS

www.orthopaper.com

Received: 21-05-2017

Accepted: 22-06-2017

Dr. Subbiah M

MS, DNB, MNAMS, FNB

(Spine Surgery), Assistant

Professor, Department of

Orthopaedics and Spine Surgery

Velammal Medical College

Hospital and Research Institute,

Velammal Village, Airport-

Mattuthavani Ring Road,

Chinna Anuppanadi, Madurai,

Tamil Nadu - 625009, India

Dr. Yegumuthu K

M.D. Assistant Professor,

Department of Pathology,

Velammal Medical College

Hospital and Research Institute,

Velammal Village, Airport-

Mattuthavani Ring Road,

Chinna Anuppanadi, Madurai,

Tamil Nadu - 625009, India
Correspondence

Dr. Subbiah M

MS, DNB, MNAMS, FNB

(Spine Surgery), Assistant

Professor, Department of

Orthopaedics and Spine Surgery

Velammal Medical College

Hospital and Research Institute,

Velammal Village, Airport-

Mattuthavani Ring Road,

Chinna Anuppanadi, Madurai,

Tamil Nadu - 625009, India

\section{A modified transforaminal lumbar interbody fusion technique to decrease the incidence of postoperative radiculopathy: A prospective analysis of 216 patients}

\author{
Dr. Subbiah M and Dr. Yegumuthu K
}

DOI: http://dx.doi.org/10.22271/ortho.2017.v3.i3i.91

\section{Abstract}

Reported incidence of postoperative intractable radiculopathy following posterior lumbar interbody fusion (PLIF) and transforaminal lumbr interbody fusion (TLIF) is between $7-14 \%$ affecting postoperative recovery. Excessive medial dural retraction in PLIF and exiting nerve root injury in TLIF during cage insertion are the proposed itiologies for battered root syndrome. Aim of the study was to identify the incidence of postoperative radiculopathy on the side of cage insertion following a modified TLIF technique. 216 patients who underwent fusion by modified-TLIF approach were prospectively analyzed. The inferior facet of cranial vertebra alone was excised retaining the pars interarticularis of cranial vertebra and superior facet of caudal vertebra which from the posterior wall of intervertebral foramen. It provided adequate space for cage insertion without need for excessive dural retraction in addition to protecting the exiting nerve root. The severity of postoperative radiculopathy on the side of cage insertion was assessed by visual analog scale (VAS). Of the 216 patients, L4-5 level was fused in 137, L5-S1 in 72 and L3-4 in 7 patients. Severe postoperative radiculopathy (VAS: 10) was observed only in three patients with an incidence of $1.4 \%$ who needed prolonged hospitalization for its recovery. L3-L4 was the fused level in these three patients while none of them in L4-5 and L5-S1 levels had such severe radiculopathy. Lumbar interbody fusion by modified TLIF (Hemi-TLIF) approach involving excision of only the inferior facet decreases the incidence of post-operative radiculopathy. However, we recommed this procedure only at L4-5 and L5-S1 levels where the spinal canal is relatively larger when compared to higher lumbar levels.

Keywords: Lumbar interbody fusion; PLIF; TLIF; Postoperative radiculopathy; Modified TLIF; Battered root syndrome; Hemi-TLIF

\section{Introduction}

Lumbar interbody fusion is commonly performed for many spinal disorders and addition of pedicle screw instrumentation improves fusion rate by providing direct vertebral stability ${ }^{[1,2]}$. Posterior lumbar interbody fusion (PLIF) and transforaminal lumbar interbody fusion (TLIF) are the two commonly performed posterior fusion techniques. The posterior wall of intervertebral formaen through which the nerve roots exits each lumbart level is formed by the superior articular facet of caudal vertebra and pars interarticularis part of the cranial lamina. Interbody cage insertion in PLIF is done by retracting the dura through the laminectomy defect without damaging facet joints while TLIF involves fusion without dural retraction by complete excision of superior and inferior articular processes of facet joint. Of all potential complications of PLIF and TLIF, persistent post-operative severe intractable radiculopathy due to "battered root syndrome" is reported in $7-14 \%$ of patients significantly affecting their early postoperative recovery necessitating further treatment ${ }^{[3,4]}$. This postoperative radiculitis is attributed to excessive medial retraction of traversing nerve root in PLIF and direct injury to exiting nerve root during cage insertion in TLIF ${ }^{[5]}$.

We analysed the incidence of postoperative radiculopathy on the side of cage insertion in lumbar interbody fusion using a single bullet cage inserted obliquely by modified TLIF approach (Hemi-TLIF). The inferior facet of cranial vertebra alone was osteotomized retaining the superior facet of caudal vertebra and pars interarticularis part of the lamina of cranial vertebra that together form the posterior wall of intervertebral foramen to prevent injury to the 
exiting nerve root in addition to providing space for cage insertion without excessive dural retraction.

\section{Materials and methods}

216 patients who underwent lumbar interbody fusion by modified TLIF approach using a single oblique bullet cage were prospectively analysed. The indications for surgery were lumbar spondylolisthesis, degenerative disc disease, failed back surgery syndrome and spondylodiscitis. All these patients underwent surgery as they were not responding to adequate trial of conservative management. Plain radiographs of lumbosacral spine and MRI were obtained in all patients preoperatively.

\subsection{Surgical procedure}

All procedures were performed by a single surgeon. The patient was postioned prone in a spinal frame under general anaesthesia after adequate padding of bony prominences. A standard posterior midline exposure of the levels to be fused was done till the lateral border pars interarticularis on both sides. Pedicle screws were inserted into the vertebrae and rods fixed. Distraction was applied between the screws to open up the disc space.

The interspinous ligament was excised and the inferior half of spinous process of cranial vertebra was nibbled till its base where it merges with the two laminae. The caudal edge of the superior lamina was removed with rongeurs till the attachment of ligamentum flavum to its anterior surface was visualized. The sagittally oriented lumbar facet joint on the side of preoperative radicular pain was exposed by excising the facet joint capsule. The inferior articular process of cranial vertebra on that side was osteotomized with a narrow osteotome starting in the midline at the level of junction between the two laminae and base of spinous process and proceeding laterally (Fig 1).

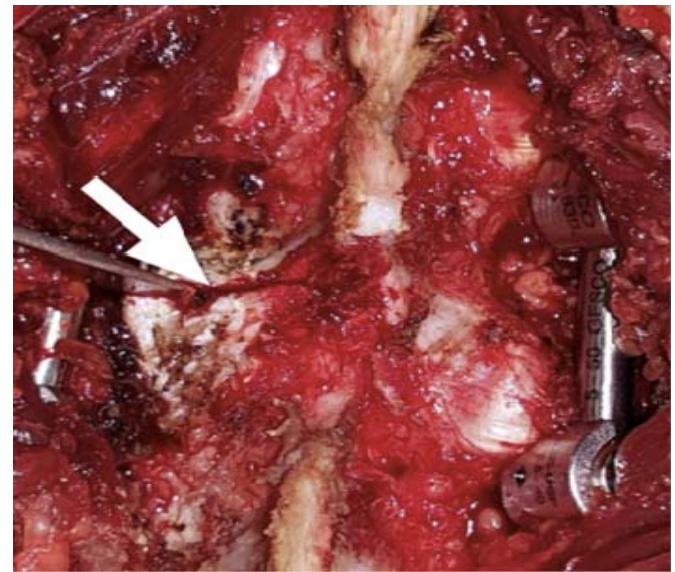

Fig 1: Intraoperative photograph showing the line of osteotomy (white arrow) of inferior articular facet.

The ligamentum flavum was retained till the osteotomy of inferior articular process was completed to prevent inadvertent entry of osteotome into spinal canal. Care was taken to prevent injury to the laterally placed superior articular process of caudal vertebra.

After excising the osteotomized inferior facet, articular cartilage of superior articular process and retained ligamentum flavum were clearly visualized (Fig 2).

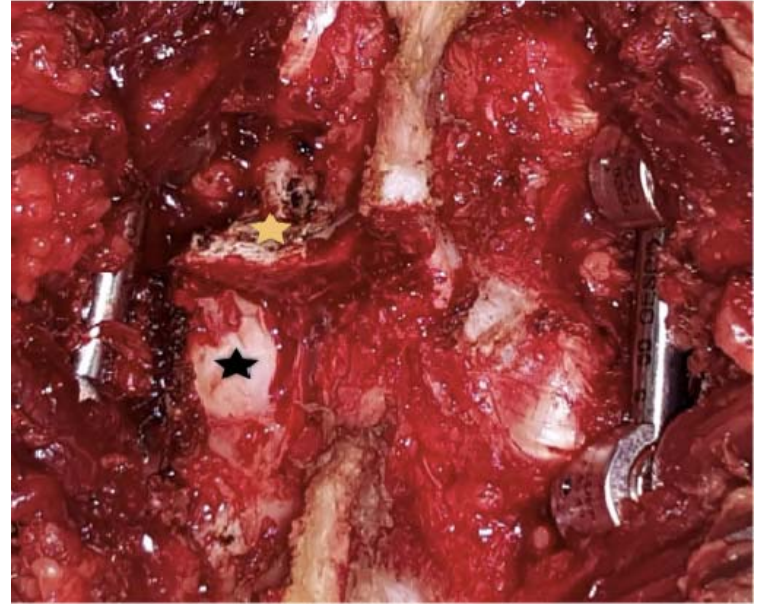

Fig 2: Intraoperative photograph after excision of inferior articular facet showing articular cartilage of preserved superior articular facet (black star) and pars-interarticularis part of cranial lamina(yellow star) which form the posterior wall of vertebral foramen protecting the nerve root.

The remaining attachment of ligamentum flavum was released from the upper border of the caudal lamina and medial border of the retained superior facet to expose the dura and traversing nerve root. The exiting nerve root above was protected by the remaining upper half of cranial lamina and the superior articular process of caudal vertebra which together form the posterior wall of vertebral foramen (Fig 2). The medial edge of the superior articular process which overhangs into the spinal canal was removed with a Kerrison rongeur to expose the disc space lateral to the dura. Discectomy was performed through this space between the dural sac and medial edge of superior articular process without retracting the traversing nerve root. Autografts from the excised spinous process and inferior articular process was packed into the disc space. A single bullet cage packed with local autografts was introduced obliquely into the disc space through the space between dura and superior articular process (Fig 3).

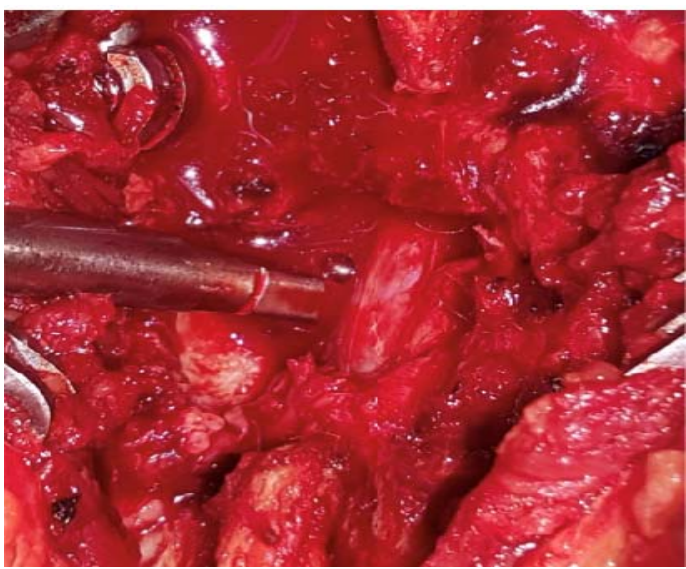

Fig 3: Intraoperative photograph showing introduction of cage through the space lateral to dural sac without dural retraction.

It was directed obliquely to be postioned in such a way that it crosses the midline in AP view (Fig 4). 


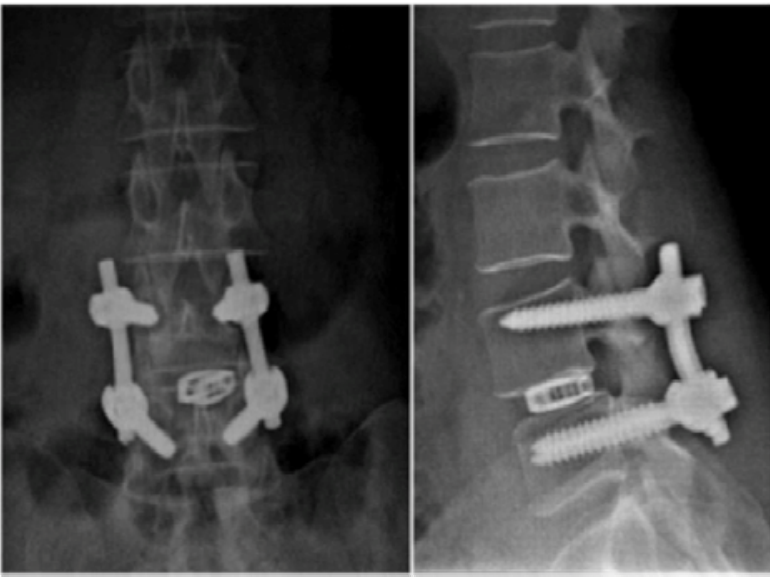

Fig 4: Postoperative anteroposterior and lateral radiograph after modified TLIF showing the position of bullet cage.

The retained cranial half of the superior lamina and the superior articular process of the caudal vertebra prevent injury to the exiting nerve root during this stage of cage insertion. The contralateral nerve root was decompressed if necessary. The pedicle screws were compressed and final tightening of the nuts done. Wound was closed in layers over a drain.

The patients were made to sit up on the first postoperative day (POD) and mobilized with a lumbosacral orthosis after drain removal on the second POD. Patients were evaluated for radicular pain in the immediate postoperative period on the side of cage insertion and when present the severity was assessed by visual analog scale (VAS) for radicular pain.

\section{Results}

The mean age of patients was 52 years (Range: 24-76 years) and mean duration of follow up was 26 months (Range:18-36 months). 142 patients were males and the remaining 74 were females. The indications for fusion were lumbar spondylolisthesis in majority of patients (n: 149), while other indications included spondylodisciitis(n-41), failed back surgery syndrome(n-19) and degenerative disc disease(n-7).

The mean operative time was 75 minutes and mean blood loss was $150 \mathrm{ml}$. The mean duration of hospital stay was 8 days (Range: 5-21 days). The commonest level fused was L4-5 (n137) while L5-S1 was fused in 72 and L3-4 in 7 patients. Titanium bullet cage was used as an interbody spacer in 174 patients and poly-ether-ether-ketone (PEEK) cage was used in 42 patients.

Severe radiculopathy on the side of cage insertion was observed only in three patients in immediate post-operative period with an incidence of $1.4 \%$. The VAS score for leg pain in these three patients was 10 and L3-L4 was the fused level in these patients. They needed prolonged hospital stay for a period of 17-21 days. However, they responded well to conservative management with steroids, opiods, NSAID's and they did not require resurgery for root decompression or rhizotomy procedures. Mild sensory disturbance in the form of paraesthesia was observed in 32 patients on the side of cage insertion $(\mathrm{VAS}<3)$ which did not affect their postoperative mobilization protocol. Superficial wound infection was observed in three patients who needed debridement and secondary closure of the wound while dural tear occurred in 9 patients.

\section{Discussion}

In addition to maintaining the load bearing capacity of spine, interbody fusion also aids in achieving sagittal spinal alignment and increases the available area of fusion bed to enhance arthrodesis ${ }^{[6]}$. Cloward in 1940 performed the first PLIF ${ }^{[7]}$ which was later modified by Lin ${ }^{[8]}$. TLIF procedure was pioneered by Harms in 1998 for spondylolisthesis, scoliosis and post-discectomy syndrome ${ }^{[9]}$. The reported incidence of complications following PLIF and TLIF is $36.4 \%$ including subsidence of cage, osteolysis, dural tear, intraoperative neurological injury etc ${ }^{[3]}$. Of these, the most disabling complication is intraoperative neurological injury resulting in "battered root syndrome" which is reported to occur in $7-10 \%$ of cases causing severe post-operative radiculopathy $[3,4]$. Moatz et al described this entity to be placing patients at increased risk even when other factors are equal when comparing TLIF/PLIF with standard PLF with instrumentation ${ }^{[3]}$. Battered root syndrome was first described by Bertrand in 1975 as a permanent radiculopathy caused by surgical trauma. He defined it as a reappearance of intractable radicular pain following the relief of sciatic pain by surgery with a constant burning character. The proposed treatment modalities for this condition when not responding to conservative management include rhizotomy, spinal cord stimulation etc. Carragee et al reported a complication rate of $10-50 \%$ including radiculitis, heterotopic ossification etc. associated with the use of bone morhogenetic protein(BMP) in PLIF and TLIF, however they were not able to correlate post-operative leg pain and heterotopic ossification [10]. Postoperative radiculitis was reported in $14 \%$ of patients who underwent TLIF with rhBMP-2 [11]. Humphreys et al attributed the need for greater retraction of dura for the high incidence of post-operative radiculitis in patients undergoing PLIF ${ }^{[5]}$. Mehta et al. reported an increased incidence of nerve root complications and durotomy following PLIF and TLIF and concluded that PLIF/TLIF should be performed only when the goals of surgery cannot be accomplished by decompression and traditional PLF ${ }^{[13]}$.

Discectomy and interbody fusion in PLIF is done by retracting the dural sheath till midline or sometimes beyond to access the disc space without removing superior or inferior articular process. This excessive dural retraction might result in traversing nerve root damage or severe postoperative neurogenic radicular pain on that side ${ }^{[14]}$. Interbody fusion in TLIF is performed through far lateral aspect of disc space after removing the superior and inferior articular process of facet joint completely. This complete facet removal exposes the obliquely coursing exiting nerve root making it vulnerable to injury and postoperative radiculopathy in TLIF occurs due to occult injury to this exiting nerve root on the far lateral aspect when cage is inserted. Postoperative radicular pain following TLIF was reported to occur in $13.8 \%$ of patients by Burneikiene et al ${ }^{[15]}$.

The posterior wall of intervertebral foramen through which the spinal nerve root exits is formed by lower portion of pars interarticularis of the lamina of cranial vertebra superiorly and superior articular process of caudal vertebra inferiorly ${ }^{[16]}$. Our procedure involving osteotomy of inferior articular process of cranial vertebra alone retains this posterior wall of vertebral foramen protecting the exiting nerve root in addition to providing necessary space for cage insertion without need for excessive dural retraction. Hence, we propose that the problems secondary to iatrogenic battered nerve root in PLIF and TLIF can be avoided by this technique of hemi-TLIF involving the resection of only the inferior articular process. Persistent postoperative severe radiculopathy due to battered nerve root syndrome was reported in $7-14 \%$ of patients affecting early recovery necessitating further treatment $[3,4,11$, 
15]. Postoperative severe radiculopathy in our series of patients was less with an incidence of only $1.4 \%$ (3/216 patients). These three cases who had severe postoperative radiculopathy in our study were found to be at L3-L4 level with which we propose this procedure to be suitable only for fusions involving the L4-L5 and L5-S1 levels where the spinal canal is relatively larger than higher lumbar levels.

\section{Conclusion}

Lumbar interbody fusion by our modified-TLIF (Hemi-TLIF) approach involving excision of inferior facet of cranial vertebra alone provides adequate space for cage insertion without the need for excessive dural retraction in addition to preventing injury to exiting nerve root by the retained posterior wall of vertberal foramen which protects it. This procedure significantly decreased the incidence of severe post-operative radiculopathy and aids in early post-operative recovery. However, we recommed this procedure only at L4-5 and L5-S1 levels where the spinal canal is relatively larger when compared to higher lumbar levels.

\section{References}

1. Hallett A, Huntley JS, Gibson JN. Foraminal stenosis and single-level degenerative disc disease: a randomized controlled trial comparing decompression with decompression and instrumented fusion. Spine (PhilaPa 1976) 2007; 32:1375-80.

2. France JC, Yaszemski MJ, Lauerman WC. A randomized prospective study of posterolateral lumbar fusion: outcomes with and without pedicle screw instrumentation. Spine (Phila Pa 1976) 1999; 24:553-60.

3. Moatz P, Tortolani PJ. Transforaminal lumbar interbody fusion and posterior lumbar interbody fusion utilizing BMP-2 in the treatment of degenerative listhesis: Neither safe nor cost effective. Surg Neurol Int: 2013; 22(4):S67-73.

4. Chrastil J, Patel AA. Complications associated with posterior and transforaminal lumbar interbody fusion. $\mathrm{J}$ Am Acad Orthop Surg. 2012; 20:283-91.

5. Humphreys SC, Hodges SD, Patwardhan AG. Comparison of posterior and transforaminal approaches to lumbar interbody fusion. Spine. 2001; 26:567-571.

6. Voor MJ, Mehta S, Wang M. Biomechanical evaluation of posterior and anterior lumbar interbody fusion techniques. J Spinal Dis 1998; 11:328-334.

7. Cloward R. The treatment of ruptured lumbar intervertebral discs by vertebral body fusion. J Neurosurg. 1953; 10:154-168.

8. Lin PM. A technical modification of Cloward's posterior lumbar interbody fusion. Neurosurgery. 1977; 1:118-124.

9. Harms JG, Jeszenszky D. [Die posteriore, lumbale, interkorporelle Fusion in unilateraler transforaminaler Technik]. Oper Orthop Traumatol 1998; 10:90-102. German.

10. Carragee EJ, Hurwitz EL, Weiner BK. A critical review of recombinant human bone morphogenetic protein-2 trials in spinal surgery: Emerging safety concerns and lessons learned. Spine J. 2011; 11:471-91.

11. Rihn JA, Patel R, Makda J, Hong J, Anderson DG, Vaccaro AR et al. Complications associated with singlelevel transforaminal lumbar interbody fusion. Spine J. 2009; 9:623-9.

12. Mehta VA. Trans-foraminal versus posterior lumbar interbody fusion. Comparison of surgical morbidity. Neurol Res. 2011; 33(1):38-42.
13. Deng-lu Yan, Fu-xing Pei, Jian Li, Cheng-long Soo. Comparative study of PILF and TLIF treatment in adult degenerative spondylolisthesis. Eur Spine J. 2008; 17:1311-1316.

14. Lumbar degenerative spinal deformity: Surgical options of PLIF, TLIF and MI-TLIF Hwee Weng Dennis Hey, Hwan Tak Hee. IJO 2010; 44(2).

15. Burneikiene S. Complications in patients undergoing combined transforaminal lumbar interbody fusion and posterior instrumentation with deformity correction for degenerative scoliosis and spinal stenosis. Surgical Neurology International 2012; 3:25.

16. Rema D, Rajagopalan N. Morphometry of lumbar intervertebral foramen. Indian J Orthop. 2005; 39:3:145147. 